\title{
Gaetano Dammacco
}

\section{I beni temporali nel diritto canonico}

Prawo Kanoniczne : kwartalnik prawno-historyczny 43/3-4, 291-321

2000

Artykuł został zdigitalizowany i opracowany do udostępnienia w internecie przez Muzeum Historii Polski w ramach prac podejmowanych na rzecz zapewnienia otwartego, powszechnego i trwałego dostępu do polskiego dorobku naukowego i kulturalnego. Artykuł jest umieszczony w kolekcji cyfrowej bazhum.muzhp.pl, gromadzącej zawartość polskich czasopism humanistycznych i społecznych.

Tekst jest udostępniony do wykorzystania w ramach dozwolonego użytku. 
Prawo Kanoniczne

43 (2000) nr 3-4

\section{GAETANO DAMMACCO}

\section{BENI TEMPORALI NEL DIRITTO CANONICO}

1- Il rinnovato assetto ecclesiologico dopo il Vaticano II come categoria giuridica interpretativa. 2- uso dei beni e insegnamento del Concilio. La distinzione in beni creati, beni materiali, beni ecclesiastici. 3- Finalità della Chiesa e diritto a possedere $\mathrm{i}$ beni: missione e comunione ecclesiale. Le relazioni tra le chiese e il ricorso alla perequazione solidale nella vita della Chiesa. 4- Le novità del Libro V del codex iuris canonici. L'attuazione dei precetti conciliari e la struttura comunitaria della Chiesa. I riflessi suHa disciplina codiciale dei beni dell'immagine di Chiesa come Popolo. 5- L'autonomia concettuale e formale del diritto patrimoniale. Il diritto a possedere. La generale capacità soggettiva. Il carattere ecclesiale dell'appartenenza e la valutazione economica dei beni. Le finalità dei beni. Le finalità del dominio sui beni. 6- La dimensione pubblicistica del diritto patrimoniale. I beni temporali e i beni ecclesiastici nel codex iuris canonici. La scelta comunitaria e il bene comune della Chiesa. 7- Le entrate patrimoniali della comunità ecclesiale. L'imposizione ordinaria dei tributi e il fine della Chiesa. Le necessità ecclesiali. La funzione delle Conferenze Episcopali. La politica patrimoniale ecclesiastica. L'imposizione straordinaria. 8- Le oblazioni e le offerte. L'offerta per la messa: peculiarità e modalità controprestativa. 9- I contratti e le alienazioni: l'esclusione dello scopo di lucro e la valenza ecclesiale. 10- L'amministrazione dei beni: le scelte conciliari e l'immagine di Chiesa. I principi direttivi circa l'amministrazione: a) la titolarità e l'ufficio di amministratore (centralità del Pontefice e del Vescovo. Il principio di sussidiarietà); b) la corresponsabilità (il Consiglio per gli affari economici, il Collegio dei consultori, il Consiglio presbiteriale, il Consiglio pastorale, l'economo); c) la complementarietà (l'Istituto di raccolta dei beni, il fondo comune); d) la solidarietà e la mutua cooperazione (gli istituti diocesani federati, la consociazione, l'alternativa al 
beneficio); e) l'ordinaria e la straordinaria amministrazione. 11- La nuova disciplina canonica dei beni ecclesiastici impone un diverso modo di stabilire a tal riguardo il rapporto con l'ordinamento civile.

1 - Le norme contenute nel libro $V$ del codex iuris canonici del 1983, che disciplinano i beni temporali della Chiesa, contengono peculiarità sostanziali, che esigono un preliminare e necessario richiamo di metodo ai precetti ed alle opzioni del Concilio Ecumenico Vaticano II. La rinnovata immagine della Chiesa, offerta e rielaborata dal Concilio, indica alcune categorie interpretative fondamentali e imprescindibili, alla luce delle quali esaminare le specifiche previsioni normative e tutte quante nel loro insieme, in un'opera di adattamento «all'assetto ecclesiologico messo in evidenza» (cfr. Lo Castro, La qualificazione giuridica delle deliberazioni conciliari, Milano, 1970, p. 33). Un rinnovato sensus ecclesiae consente di rendere concreto il legame, già indicato da Giovanni Paolo Il nella Costituzione Apostolica $\mathrm{Sa}$ crae disciplinae leges, tra codice e Concilio, utilizzando le norme codiciali non in una prospettiva volta a delimitare le differenti posizioni individuali, bensì evidenziando il carattere di complementarietà esistente tra codice e Concilio e di generale dinamicità ravvisabile tra diritto e vita della Chiesa.

L'accostamento al nuovo codice, che non può essere considerato come espressione di un semplice processo di adeguamento di quello piano-benedettino alle mutate esigenze religiose e sociali delle comunità ecclesiali ma come nuova disciplina derivata da una evoluzione generale e diffusa della cristianità e della Chiesa cattolica, esige una mentalità rinnovata e, al contempo, nuove tecniche interpretative, prevalentemente orientate da una conoscenza generale dell'evoluzione della scienza ecclesiastica e dalla acquisizione dei progressi attraverso i quali si manifesta l'ortoprassi ecclesiale. Per questo, l'esame specifico delle norme che disciplinano la materia patrimoniale deve essere necessariamente preceduta dal rinvio all'insegnamento del Concilio, il quale, dunque, assume carattere vincolante sotto un duplice aspetto: uno di natura etica e di carattere strettamente teologico; ed un secondo di carattere direttamente giuridico, per la natura cogente dei precetti conciliari.

2 - Con specifico riferimento alla materia dei beni materiali è interessante notare come il Concilio non offra una trattazione sistematica 
e una definizione dell'assetto patrimoniale della Chiesa. A questa scelta del legislatore del Concilio sembra, ma solo apparentemente, far da contrasto la regolamentazione con carattere di autonomia adottata dal legislatore codiciale.

I Padri conciliari hanno ritenuto più conveniente trattare la subiecta materia, quando se ne presentavano la necessità e l'occasione, con riguardo al complesso delle problematiche concernenti l'assetto interno della comunità ecclesiale e quello dei rapporti con la società civile afferente il più vasto ambito della testimonianza della comunità ecclesiale.

Tuttavia, analizzando nell'insieme i documenti conciliari nei brani nei quali si tratta dei beni temporali (segnatamente si possono segnalare, tra gli altri, Lumen gentium n. 36, Christus Dominus n. 12, Gaudium et spes nn. 69-70-71, Apostolicam actuositatem n. 7, Presbyterorum Ordinis nn. 8-17) anche con riguardo alle opzioni ed ai ,consigli" generali emergenti, è possibile ricavare una serie di indicazioni, alle quali si può attribuire un valore ora etico, ora teologico, ora pedagogico, ora giuridico.

Preliminare, per il valore pedagogico riferito al contenuto teologico interpretativo dell'evento della creazione, è la distinzione dei beni temporali in: a) beni creati, b) beni materiali, c) beni ecclesiastici.

a) Il tema concernente i beni creati viene affrontato già ne! par. 36 della costituzione conciliare Lumen gentium, nella parte relativa al capo IV circa i laici, con più diretto riferimento alla dignità regale di questa componente del popolo di Dio ed all'opera di cooperazione che i laici sono chiamati a realizzare con riferimento al „fatto" della creazione, intesa sia come origine delle cose sia come alleanza con Dio in Cristo per il raggiungimento del fine dell'umanità, attraverso la realizzazione della giustizia, della carità, della pace. In questa prospettiva di alleanza con l'umanità, i beni creati sono posti in diretta relazione con il lavoro dell'uomo, con il progresso tecnico e con quello culturale e sono caratterizzati dall'essere posti per l'utilità comune di tutti quanti gli uomini, affinché essi siano più giustamente distribuiti e $<$ contribuiscano al progresso universale nella libertà umana e cristiana $>$ (Lumen gentium, n. 36). $<<$ Il possesso dei beni materiali, il loro sviluppo e la loro giusta distribuzione $\gg$ (vedi sul punto decreto sull'ufficio pastorale dei Vescovi nella Chiesa, Christus Dominus, n. 12), poiché in qualche misura costituiscono una partecipazione all'amicizia di Dio verso l'uomo, implicano un impegno dottrinale ed impongono ai Vescovi l'esercizio del munus docendi. 
b) Conseguentemente, $\mathrm{i}$ beni materiali, che possono essere individuati in quelle realtà <<che costituiscono l'ordine temporale, cioè i beni della vita e della famiglia, la cultura, l'economia, le arti e le professioni, le istituzioni della comunità politica > e per questo dotate di "fine proprio", ancorché poste in relazione al fine ultimo dell'uomo (cfr. decreto sull'apostolato dei laici, Apostolicam actuositatem, $n$. 7), sono caratterizzati dalla destinazione comune, sia quando l'appartenenza è individuale sia nella ipotesi di appartenenza pubblica (cfr. la costituzione pastorale Gaudium et spes, nn. 69, 70, 71). Sicché, il retto uso dei beni economici, cioè di quelle realtà temporali afferenti l'ambito dell'economia, questione alla quale il Concilio riconosce carattere di urgenza tra i problemi del mondo contemporaneo meritevoli di immediata trattazione, proprio perché caratterizzato $<$ da un crescente dominio dell'uomo sulla natura, dalla moltiplicazione e dalla intensificazione dei rapporti e dalla interdipendenza tra i cittadini, gruppi e popoli, come pure da un più intenso intervento dei pubblici poteri $\gg$ (Gaudium et spes n. 63), determina effetti sia nel campo, per così dire, esterno della convivenza umana, sia nel campo interno della vita ecclesiale, nella quale l'ordine temporale costituisce una via per la santità e la perfezione dello stato di vita dei fedeli ( $\mathrm{Lu}$ men gentium n. 42). In questa linea teologica, la peculiare vocazione dei presbiteri è caratterizzata da una differente tensione alla comunione dei beni in un'ottica di preferenza dello spirito della fraternità, in adesione ad un sano principio di povero distacco per un pronto esercizio del ministero (confronta il decreto sul ministero e la vita sacerdotale, Presbyterorum Ordinis, nn. 8, 17),

c) I beni ecclesiastici propriamente detti, che costituiscono una specificazione dei beni temporali, sono quelli direttamente finalizzati al raggiungimento degli scopi della Chiesa circa la sistemazione del culto divino, il dignitoso mantenimento del clero, il sostenimento delle opere di carità e di apostolato, specie a beneficio dei poveri (Presbyterorum Ordinis n. 17).

L'appartenenza di taluni beni alla comunità ecclesiale, quindi, è direttamente ed esclusivamente connessa con detta triplice finalità e, nell'ambito della missione della Chiesa, riveste un peculiare carattere di strumentalità. Per questo la disciplina codiciale, nel dare veste normativa ai menzionati principi di diritto, nel can. 1257 definisce bona ecclesiastica $<<$ solamente quelli appartenenti alla Ecclesia universa $>$, alla S. Sede e alle altre $<<$ personae iuridicae publicae $>>$. In tal 
modo, quindi, si attribuisce un'importante conseguenza di ordine pratico a quella distinzione tra ,personae iuridicae" che forma oggetto del can. 116 (confronta sul punto T.MAURO, Gli aspetti patrimoniali dell'organizzazione ecclesiastica, in Il nuovo codice di diritto canoni$c o$, a cura di S. FERRARI, Bologna, 1983, 208). Si comprende, allora, la ratio conciliare che governa la disciplina giuridica alla quale sono sottoposti i bona ecclesiastica, diretta espressione di una teologia più genuina e più vicina allo spirito evangelico, e perché il legislatore canonico abbia previsto una proibizione di carattere generale riguardante il divieto assoluto di accumulo della ricchezza e un corrispondente onere, anch'esso di carattere generale, di attenzione continua ai poveri e al bene comune della Chiesa e alle opere di carità.

3 - In questa dimensione di strumentalità si coglie in tutta evidenza il primato della missione della Chiesa, che consiste nell'ordinare a Dio ogni realtà temporale e nel privilegiare, sempre e comunque, la persona umana, centro dell'azione_redentiva divina (confronta l'enciclica Redemptor hominis).

Questo modo rinnovato di concepire il rapporto con i beni economici, al quale non può risultare estraneo il rinnovamento delle relazioni che il cristiano stabilisce con tutte le realtà del mondo, determina un duplice effetto sulle relazioni interne della comunità ecclesiale, esaltando due momenti della comunione e stabilendo in tal modo alcuni principi di diritto, come ad esempio a) la necessità della perequazione tra le Chiese, come aspetto della relazione intraecclesiale, e b) la necessità della perequazione interna ad ogni Chiesa, come manifestazione della comunione intersoggettiva.

a) La sollecitudine per la Chiesa universale, posta nella Lumen gentium al n. 23 come un effetto della riscoperta dell'autonomia delle Chiese locali e quale manifestazione dell'appartenenza dei singoli vescovi al Collegio episcopale e peculiarità dell'esercizio del munus docendi, costituisce un aspetto del tutto nuovo dell'universalità e dell'unità della fede nel popolo di Dio. In modo peculiare il rapporto tra il collegio dei pastori, i compiti di solidarietà dei fedeli e la pluralità delle Chiese si traduce in una potestas regiminis nell'esercizio di un dovere di solidarietà. Questa solidarietà è espressione della comunione universale della carità, che si realizza non solo nei confronti delle missioni, ma anche nei confronti delle altre Chiese più povere e disagiate. In quest'opera di solidarietà non è impegnato solo il vescovo a titolo 
personale, ma in quanto capo di una comunità ecclesiale particolare, sicché l'esercizio solidale della carità e della cooperazione nel mutuo scambio di doni, di beni e di energie si trasferisce a tutti i fedeli, e non a circoli limitati del clero e del laicato, quale peculiare derivazione dell'universalità del popolo di Dio (Lumen gentium n. 13). Il dovere di solidarietà, quindi, costituisce un principio di diritto che trova la sua adeguata formulazione normativa non solo nel canone 111, par. 1, dove si stabilisce l'incorporazione alla Chiesa con il battesimo, ma in modo specifico nel canone 209, che detta un obbligo di carattere generale circa la conservazione della comunione con la Chiesa attraverso l'adempimento di doveri nei confronti della Chiesa universale e di quella particolare.

b) Per quanto riguarda la perequazione interna, in questa sede converrà sottolineare la duplice caratteristica individuata dai precetti conciliari e ripresa successivamente anche nella Ecclesiae sanctae, art. 8. Alla luce di questa disposizione, si deve rigettare l'ipotesi di trasformazione del problema del sostentamento del clero in una specie di rivendicazione dei diritti di una categoria, poiché Presbyterorum Ordinis al n. 20 stabilisce per i fedeli un obbligo a provvedere (<<vera obbligatione tenentur $>>$ ) attribuendo a detta obbligazione valore ecclesiale.

Una seconda caratteristica consiste nell'indicare i valori della solidarietà, della fraternità e dell'amicizia tra tutti i presbiteri, come beni ecclesiali che attraversano l'esistenza quotidiana e danno forma ad uno stile di vita concreta (Presbyterorum Ordinis nn. 17, 20, 21).

Questa duplice caratteristica anima l'insieme delle norme che nel codice di diritto canonico disciplinano la materia del sostentamento del clero e, pertanto, insieme ad altre specifiche previsioni, come ad esempio quella contenuta nel canone 281 , rende esplicita la ratio teologica di una Chiesa che vive nella sua prassi la verità evangelica attraverso la forma giuridica.

4 - Il riferimento ai precetti ed ai principi conciliari, ancorché sommario e tuttavia essenziale per la comprensione delle novità sostanziali che caratterizzano la disciplina del codice circa $i$ beni temporali della Chiesa, consente di avvicinarsi con più coerenza all'interpretazione del contenuto delle singole norme e alla valutazione di tutte nel loro insieme, opera necessaria soprattutto se si considera che la materia in taluni punti formalmente pare riprodurre la disciplina del vecchio codice pianobenedettino. Occorre, tuttavia, avvertire che 
l'eguaglianza normativa è solo ed esclusivamente apparente, poiché la previsione normativa, anche quando pare riprodurre il dettato del vecchio codex, ha vita e contenuto differenti, così come differente è il rapporto con il mutato quadro teologico di riferimento.

Una prima differenza emerge dall'osservazione circa la collocazione sistematica della materia, la quale viene autonomamente ordinata nel libro $V$ laddove nella vecchia disciplina codiciale trovava ospitalità nella Pars sexta del Liber tertius. La disciplina sui beni è in modo strumentale posta dopo i libri riguardanti il Popolo di Dio il Munus docendi e il Munus sanctificandi. Questa peculiare sistemazione pone un primo criterio circa il metodo interpretativo e suggerisce di procedere nell'esame della normativa in modo libero da rigide costruzioni dogmatiche, fondate su una logica obsoleta, più attenti alla ricerca di una oggettiva autonomia concettuale fondata su una ratio unitaria che, derivata dai precetti e dalle opzioni del Concilio, prende corpo e organicità nei libri precedenti. La autonomia del libro $V$ comporta una analisi della materia ispirata ad una organicità interna e propria, che comunque va raccordata con il processo di relativizzazione che caratterizza il rinnovamento del sistema giuridico canonico, dal quale la materia trae significato e ratio. Questo processo di relativizzazione, che cerca di evidenziare le interconnessioni esistenti tra teologia, diritto e concilio (approfondendo gli aspetti magisteriali, storici, filosofici, sociologici, pastorali, eccetera), non assolutizza le singole scienze ma, rendendole tra loro complementari, ne caratterizza la più intrinseca dinamicità e autonomia, mettendo a nudo le aporie e le lacune del sistema nell'intento di superarle. In questa logica, centrale è l'immagine che, sull'insegnamento del Concilio, il legislatore codiciale ha posto a fondamento della conoscenza dell'esistenza storica ed attuale della Chiesa, cioè l'immagine di Popolo di Dio.

Detta immagine non è la sola indicata come idonea a comprendere l'esistenza storica e misterica della comunità ecclesiale nella società e nel mondo, tuttavia è quella privilegiata. Ciò che consente di meglio comprendere la realtà alla quale si riferisce, rendendola storica ed attuale, pur non ignorando i collaterali effetti di riduttività. L'uso dell'immagine non è il modo esclusivo della conoscenza, ma è in ogni caso il criterio guida, alla luce del quale compiere l'opera di interpretazione delle norme giuridiche.

L'unità sostanziale del codice di diritto canonico, dominata dal prevalere dell'immagine di popolo, finisce con il riflettersi nel libro $\mathrm{V}$, 
nel quale la disciplina giuridica risponde nella forma e nella sostanza alla fondamentale domanda circa la relazione esistente tra detta immagine e il regime dei beni temporali della Chiesa.

Il popolo non è unità indifferenziata e massificata, bensi unità di elementi differenziati (e relazionati tra loro) da rapporti interattivi di complementarietà e da comportamenti disciplinati da norme strutturate intorno a bisogni comuni socialmente rilevanti. Presiede alla vita del popolo la prospettiva di una diffusa espressione di ministerialità, che secondo il messaggio evangelico caratterizza il comune status christifidelium e lo orienta verso una tensione reale e quotidiana alla comunione nel vincolo della complementarietà, della corresponsabilità e della testimonianza. Ciò in riferimento ai beni temporali determina la strutturazione dei rapporti intersoggettivi finalizzati all'uso dei beni economici, in modo da far emergere sempre il legame di comunione.

A tal proposito, si deve osservare che l'immagine di popolo è al contempo frutto di una svolta teologica verificatasi nella ecclesiologia tendente ad interpretare la Chiesa come comunità. Il prevalere di questa prospettiva si colloca nello sforzo orientato alla ricerca di una categoria interpretativa, che riveli non solo l'essenza e la natura della Chiesa, ma altresì la struttura storica, le norme che ne disciplinano l'esistenza ed i mezzi più idonei utilizzati per il perseguimento del fine che la stessa Chiesa, come fenomeno esistenziale spontaneo conseguente alla comunicazione dell'evento della salvezza operata da Cristo, si propone.

La categoria della Chiesa-comunità, secondo l'orientamento della ecclesiologia conciliare, consente di cogliere la ratio di tutto ciò che è essenziale alla natura della Chiesa, nella riscoperta della produttività degli elementi che caratterizzano detta costruzione teologica: e cioè da un lato la dimensione intersoggettiva, che conferisce valore all'elemento personale, dall'altro la natura oggettiva dell'annunzio, che giustifica la comunione ecclesiale e il complesso delle relazioni comuni nella concretezza dell'esistenza.

La categoria della Chiesa-comunità sposta l'attenzione e l'indagine ermeneutica del giurista dalla centralità delle formalità delle strutture normative alla concretezza della sostanza del rapporto interpersonale di comunione, che fonda l'intimo interesse reciproco nelle quotidiane vicende della comunità cristiana.

Pertanto, al fine di una corretta interpretazione dei canoni che nel libro $V$ disciplinano i beni temporali della Chiesa (esigenza che trova 
fondamento nel più generale bisogno di ricomprensione dell'esistenza del diritto nella Chiesa) essenziale e imprescindibile diventa il richiamo a detto quadro teologico di riferimento, dominato dal prevalere di una dimensione di Chiesa, concepita come comunità del più ampio popolo di Dio. Invero, in questa prospettiva si comprende la necessità che nella vita della comunità ecclesiale emergano i valori della solidarietà, della fraternità e dell'amicizia, più volte richiamati o presupposti dalla normativa del codice di diritto canonico. D'altra parte, viene ad evidenza il diretto richiamo del quadro teologico sopra richiamato, cioè alle categorie della comunità e del popolo, nelle norme del libro II relative ai fedeli e al titolo nel quale si tratta dei loro diritti ed obblighi (in particolare si possono indicare i cann. 204, 205, 208, 209, 223),

L'uso dei beni temporali, ed in specie di quelli economici, nei confronti dei quali non va neanche trascurata una necessaria componente pedagogica, è finalizzato ad un intento pastorale, riferito alla manifestazione della Verità e alla raffigurazione di una Chiesa più autentica e fedele al Vangelo, poiché emerge il carattere peculiarmente strumentale all'apostolato ed alla missione. In tal modo trae spiegazione il disposto del can. 1254 pr. 2, nel quale si stabilisce la triplice finalità dei beni ecclesiastici (l'ordinazione al culto divino, l'onesto sostentamento del clero e degli altri ministri, l'esercizio di opere di apostolato e di carità), in ordine alla quale si giustifica il diritto nativo della Chiesa di acquistare, possedere, amministrare, alienare i beni temporali.

In questa prospettiva è opportuno sottolineare la rilevanza generale dei canoni del L. II, Pars I, Títulus I, che, pertanto, assumono valore anche ermeneutico per l'individuazione degli schemi <<entro i quali possono e devono essere inquadrate le anzidette attività > (T. MAURO, Gli aspetti patrimoniali, cit., 209).

In particolare, va evidenziato lo stretto legame esistente tra il can. 222 e il can. 1254 pr. 2, di modo che risulta agevole comprendere come l'uso ed il possesso dei beni temporali da parte della Chiesa e l'obbligo generale da parte dei fedeli al sostegno economico sono diretti all'indicata triplice finalità e risultano motivati dalla peculiarità del legame intersoggettivo di comunione.

5 - L'intento innovatore, che si riscontra nella disciplina riservata dal codice di diritto canonico alla materia dei beni temporali, è espresso anche nella peculiare struttura formale del libro $\mathrm{V}$, che viene introdotto da cinque canoni (dal 1254 al 1258), ordinati a modo di 
premessa e di introduzione ai titoli nei quali si divide il libro. Questa caratteristica, che per la verità risulta comune a tutti i libri del codex, deriva dalla autonomia concettuale e formale che il legislatore riconosce alle singole materie dei vari libri e risponde, probabilmente, alla volontà di collocare dette materie in un quadro comune di riferimento, specificamente individuato per singola materia.

Questi canoni introduttivi raccolgono principi di carattere generale e fondamentale, che hanno lo scopo di evidenziare la ratio del sistema in un'ottica comune.

Il primo principio stabilito nel can. 1254 , pr. 1 , ancorché non sempre pacifico in un passato remoto della vita della Chiesa, è il diritto a possedere, acquistare, amministrare, alienare i beni temporali, affermato come afferente l'esistenza stessa della comunità ecclesiale e sorto con la sua istituzione. Questa capacità generale, esterna e oggettiva e per questo non dipendente dal potere civile, già riaffermata nella Dichiarazione conciliare Dignitatis Humanae n. 4, è riconosciuta in quanto la Chiesa si concepisce anche come soggetto di diritto, ed è finalizzata al perseguimento dei fini propri. Essi, in via principale, ma non esclusiva, vengono indicati nel par. 2 del medesimo can. 1254, e cioè consistono nell'ordinare il culto divino, nel provvedere all'onesto sostentamento del clero e dei ministri, nell'esercitare opere di apostolato e di carità. Si tratta di finalità che in realtà si riferiscono all'intero spettro di attività della Chiesa, la quale intende solo attuare la missione di salvezza, risultando difficile individuare altre finalità proprie rispetto alle tre contenute in detto canone. Sicché, questo secondo paragrafo, che riproduce il contenuto e la lettera del n. 17 di Presbyterorum Ordinis, probabilmente non ha solo il significato di offrire elencazioni, bensì suggerisce il richiamo metodologico al Concilio, di cui il codice vuole essere attuazione, e, al contempo, sottolinea il primato che, comunque anche in riferimento alla disciplina dei beni temporali, ha nel diritto canonico la Missione, che orienta e giustifica anche l'attività patrimoniale.

Un secondo principio consiste nell'affermazione di una generale capacità soggettiva interna, di carattere non personale, contenuto nel can. 1255. Pur considerando che il Liber Vè strutturato con riferimento alla dimensione organizzativa comunitaria della Chiesa e, pertanto, riferito alle persone giuridiche, tuttavia non può escludersi l'applicazione della ratio che lo anima anche al diritto patrimoniale riguardante le persone fisiche, indipendentemente dalle specifiche previsioni 
che nello stesso Libro si riferiscono ai singoli christifideles. Pertanto, non si può escludere che alla luce del principio affermato nel can. 1254 pr. 2 si debba interpretare anche la disposizione del can. 98 pr. 1 (che riconosce alla persona maggiorenne il pieno esercizio dei suoi diritti), sia pure nella definita accezione contenuta nel can. 222 pr. 1 (e cioè limitatamente all'obbligo di sovvenire alle necessità della Chiesa), richiamata anche nel can. 1262, che dispone sulle contribuzioni dei fedeli alle necessità della Chiesa. Interpretando queste previsioni in un'ottica comune e alla luce del principio del can. 1254 , si può riconoscere l'esistenza di un diritto di proprietà con valenza ecclesiale, e cioè connotato dal vincolo della comunione, riferito sia alle persone giuridiche come anche a quelle fisiche.

In questa prospettiva sembra debba essere letta la previsione contenuta nel can. 1256, che pur affermando il nesso di appartenenza esistente tra i beni e la proprietà della persona giuridica che li ha acquistati, sottolinea, in ogni caso, il carattere ecclesiale dell'appartenenza nel riferimento all'autorità del Romano Pontefice, nel quale a mente anche nel can. 331 e in specie del can. 1273, è riconosciuto un potere-dovere generale di tutela e di vigilanza nell' interesse della comunità ecclesiale.

Il can. 1257 pr. 1, riprendendo il contenuto del can. 1497 del vecchio codex, introduce i criteri caratteristici e distintivi circa il concetto di bona ecclesiastica. Questi non solo costituiscono quella specie dei beni materiali suscettibili di valutazione economica, che dunque ne caratterizza la natura, ma si connotano altresì per il concorrente elemento dell'appartenenza. Sicché, i due elementi concorrenti e insostituibili della economicità e dell'appartenenza (alla Chiesa, alla Sede Apostolica e alle altre persone giuridiche pubbliche, cioè alla struttura organizzativa della comunità ecclesiale) consentono di individuare quei bona temporalia sottoposti alla disciplina codiciale. L'importanza dell'elemento concorrente dell'appartenenza risulta anche dalla previsione del can. 1258 , che stabilisce un rapporto di identificazione tra la Chiesa e la struttura organizzativa di carattere pubblico. Questa disposizione, di ampio contenuto, rinvia al can. 116 (riguardante il concetto di persona giuridica pubblica), consentendo di sottolineare anche un'altra relazione esistente, in via diretta e presuntiva, tra attività delle persone giuridiche pubbliche e bene comune e/o pubblico della comunità ecclesiale. Del resto una significativa attuazione di detto principio, in virtù del quale la Chiesa nell'uso dei beni temporali per 
il tramite della struttura organizzativa comunitaria impegna sempre la propria immagine di comunità costituita in persona morale „ex ipsa ordinatione divina" (can. 113) chiamata ad attuare la missione di salvezza nel mondo (can. 204 pr. 1), riposa nel can. 1282, laddove si stabilisce che le attività compiute nell'amministrazione dei beni vanno riferite direttamente alla Chiesa, nel cui nome adempiono i propri compiti gli amministratori e coloro che a titolo legittimo hanno parte nell'amministrazione dei bona ecclesiastica.

In ogni caso, da questa ottica di ecclesialità e di pubblicità non restano escluse le persone giuridiche private e i beni dei christifideles uti singoli. Invero, il can. 1257 pr. 2, pur stabilendo l'inapplicabilità della disciplina dettata nel Libro $\mathrm{V}$ ai beni delle persone giuridiche private, contempla la possibilità di una ipotesi inversa espressamente stabilita, <<nisi expresse aliud caveatur $>$. Sicché, la previsione normativa non costituisce una norma di chiusura, ma, nell'indicare una condizione imprescindibile di carattere generale, direttamente derivata dalla Missione, individua ipotesi di elasticità, poiché 1'insopprimibile e sempre emergente finalità dell'ordinamento, cioè la salus animarum, connotando ogni aspetto della ortoprassi ecclesiale, consente di inserire in un quadro generale di finalità i beni economici $<<$ anche quando hanno una vita che si potrebbe dire d'aspetto privatistico $>$ (V. DEL GIUDICE, voce Beni ecclesiastici, in Enc. dir., Milano, 1959, V, 207).

L'analisi delle indicate disposizioni, premesse al Titulus I del Liber $V$, consente di affermare che, per la individuazione dei bona ecclesiastica, diventa necessario non solo il criterio della economicità, connesso con quello determinante dell'appartenenza, bensì anche l'ulteriore criterio del perseguimento della triplice finalità indicata nel can. 1254 pr. 2, secondo una relazione di inscindibilità tra gli stessi fini.

Infatti, l'importanza centrale delle finalità che giustificano e fondano il diritto patrimoniale della comunità cristiana, secondo quanto stabilito nel can. 1254 pr. 2, non consente di immaginare, nel contesto logico della disciplina de qua, l'esistenza di beni economici ecclesiastici, cioè appartenenti a persone giuridiche pubbliche, svincolati dall'obbligo di annunciare il Vangelo, stabilito per i fedeli nel can. 211.

Interessante, infine, sarebbe riesaminare il criterio della titolarità dei beni in relazione alla loro utilità comune ed ecclesiale, rapportandolo al fenomeno di tutte quelle comunità ecclesiali (come ad esempio le comunità c.d. di base e i movimenti ecclesiali), nelle quali il rapporto 
interpersonale costituisce in maniera più accentuata il mezzo prioritario con il quale rendere più espressivo il vincolo di comunione e dare dimensione all'appartenenza ecclesiale. In queste peculiari esperienze comunitarie il cammino di maturazione della fede è proporzionale alla crescita del senso della comunità, cosa che contrasta con l'anonimato religioso. Tuttavia, questa dimensione di comunità, più articolata verso una ampia partecipazione di base alla vita della Chiesa, non sembra trovare adeguata espressione nella disciplina codiciale che si esamina, pur riconoscendo che l'ordinamento canonico nel suo complesso apre nuove prospettive alla tematica dell'esistenza delle comunità c.d. di base (e a quelle assimilabili) e indica nuove strade. Del resto, questo tipo di esperienza comunitaria pone, senza adeguatamente risolverlo, il problema dell'equilibrato rapporto tra il carattere dell'aggregazione spontanea e interpersonale, intorno all'annuncio di salvezza, e il ruolo dell'Autorità ecclesiastica, alla quale spetta il compito di certificare l'oggettività dell'annuncio evangelico.

6 - La dimensione pubblicistica del diritto patrimoniale della Chiesa è principio che anima la disciplina contenuta nel codice di diritto canonico, anche in riferimento alla generale impossibilità di separare rigidamente nell'ordinamento canonico l'ambito del privato da quello del pubblico. In questa prospettiva va analizzata la normativa raccolta nei quattro titoli del Liber $V$, che, a mente del disposto del can. 1257 pr. 1, parrebbe disciplinare in modo specifico e diretto i beni ecclesiastici. Invero, raffrontando il dettato dell'indicato canone con la intestazione posta a capo del Libro "De bonis ecclesiae temporalibus" emerge il rapporto esistente tra i beni temporali e quelli ecclesiastici, i quali costituiscono una species del più ampio genus al quale appartengono e possono essere definiti come quelle res aventi valore economico ovvero idonee ad apportare benefici economici e finalizzate al culto, al sostentamento del clero e alle opere di carità, in particolare a beneficio dei poveri. L'indicata distinzione, comunque, emerge continuamente nel corso del Libro, che non disciplina solo i beni ecclesiastici: cosi, ad esempio, mentre il Titolo II, circa l'amministrazione dei beni, e il Titolo III, circa i contratti e le alienazioni, fanno prevalente, se non quasi esclusivo, riferimento ai beni ecclesiastici, i Titoli $I$, circa l'acquisto dei beni, e $I V$, circa le pie volontà e le pie fondazioni, disciplinano anche il vasto genere dei beni temporali. Al di là di argomentazioni, pure razionali ancorché insufficienti a spiegare le ri- 
levate diversità di regime - come ad esempio quella relativa al contenuto dei cann. 1259, 1260, 1261 per i quali può ben dirsi che essi sono posti per l'affermazione di diritti e la disciplina di quei beni temporali che, entrati poi nel patrimonio della Chiesa, diventano ecclesiastici e, quindi, sottoposti alla peculiare disciplina - evidenti si manifestano le aporie di un sistema (naturalmente complesso per la delicatezza e instabilità di una materia afferente l'ambito del temporale e perciò sempre soggetta a contrastanti giudizi), il quale guarda ad una struttura teologica ed ecclesiologica, propria di una organizzazione ecclesiastica proiettata nel futuro, mentre disciplina l'esistente, che soffre ancora i condizionamenti della passata esperienza.

L'importanza pubblicistica della disciplina circa i beni temporali, al fine di rendere più fedelmente la dimensione comunitaria della Chiesa, avrebbe richiesto, forse, una più precisa attenzione del legislatore verso l'ampio articolarsi dell'agire umano, sia individuale che sociale, giuridicamente strutturato per il conseguimento del bene ecclesiale comune. Ciò nonostante il legislatore ha preferito intitolare il libro V „De bonis Ecclesiae temporalibus”, preferendo questa formulazione all'altra „De iure patrimoniali Ecclesiae”, proposta dal "Coetus studiorum de iure patrimoniali Ecclesiae” della „Pontificia Commissio Codici iuris canonici recognoscendo", molto probabilmente perché la formulazione scelta risulta più ampiamente e direttamente riferibile al genus esteso dell'ordine temporale e più facilmente comprensibile nell'ottica della strumentalità dei beni temporali alla vita della Chiesa. Sicché, sarebbe stato preferibile evidenziare meglio e di più il legame esistente tra beni temporali, struttura comunitaria dell'agire dei fedeli e bene comune della Chiesa, rispetto a quello più chiaramente emergente nel codex e riguardante il rapporto tra beni temporali, organizzazione ecclesiastica e principi dell'attività patrimoniale della Chiesa. Una conseguenza di questa opzione, che in ogni caso mostra la caratteristiche di un fenomeno di transizione, è la scarsa applicazione di taluni principi conciliari quali la sussidiarietà e l'autonomia della Chiesa locale. Un più chiaro rimando alla struttura di comunione avrebbe potuto meglio orientare il legislatore codiciale verso una disciplina generale dell'uso dei beni, estendendo più manifestamente il concetto di dominio sui beni temporali, e quindi su quelli ecclesiastici, finalizzandone la proprietà e la disponibilità ai fini indicati nel can. 1254 pr. 2, che costituiscono il contenuto del bene comune della Chiesa. Così, in una più decisa prospettiva comunitaria, 
che nondimeno è precisa caratteristica delle norme del Liber $V$, si sarebbe potuto leggere la peculiare tutela gerarchica riferita al Romano Pontefice, nei cann. 1256 e 1273 (i quali disciplinano il medesimo principio sottolineando il primo il peso della autorità pontificia circa ogni aspetto coinvolgente l'esistenza dei beni temporali ed il secondo l'attuazione del primato di governo in riferimento all'amministrazione e alle generali condizioni di politica economica nell'uso dei beni ecclesiastici), e all'Ordinario, diocesano e non diocesano, prevista nel can. 1276. Nella prospettiva di una rinnovata rilevanza della struttura di comunione della comunità ecclesiale, si deve considerare il legame esistente tra il can. 222 (collocato nel Titolo II della Parte I del Libro II, circa i diritti e gli obblighi dei fedeli e disciplinante l'obbligo generale di sovvenire alle necessità della Chiesa per il culto divino, le opere di carità e apostolato e l'onesto sostentamento del clero) ed il can. 1254 pr. 2 (che rapporta agli stessi fini il diritto nativo della Chiesa di possedere, amministrare, acquistare, alienare i beni temporali).

7 - Il permanere della opzione comunitaria emerge anche nel Titullus $l$, relativo all'acquisto dei beni, che disciplina sostanzialmente e prevalentemente quell'acquisto che, tradizionalmente, viene definito di diritto pubblico. Le entrate patrimoniali fondate su detto titolo rispondono a due principi generali, che vanno congiuntamente esaminati, anche al fine di evitare errori di interpretazione, derivanti da un processo di sdoppiamento tra la Chiesa e i fedeli. Il primo principio è posto nel can. 1260 , che sancisce il diritto nativo della Chiesa di imporre tributi: solo in una prospettiva comunitaria è comprensibile l'affermazione che il diritto impositivo deriva alla Chiesa per costituzione, e già dalla sua istituzione, e comporta una potestas $<<$ in forza della supremitas, o meglio della potestas iurisdictionis che la Chiesa, e cioè gli organi cui spetta tale potestà, esercitano sui fedeli >> (V. DEL GIUDICE, Nozioni di diritto canonico, Milano, 1970, 430).

L'imposizione di cui si tratta, per la natura stessa del diritto riconosciuto dalla norma, non è relazionata ad alcuna offerta di servizio o di prestazione ecclesiastica, ma è direttamente riferita alle necessità per il conseguimento del fine stesso della Chiesa, $\mathrm{e}$ in specie di quelle finalità richiamate nell'art. 1254 p r. 2.

Il secondo principio è contenuto nelle disposizioni previste nei cann. 1261 e 1262 ed è direttamente connesso con il precedente: l'obbligo dei fedeli, i quali compongono la comunità ecclesiale, di contri- 
buire con sovvenzioni alle necessità della Chiesa connesso con il diritto di devolvere a favore della Chiesa i beni temporali. Quest'ultima previsione, contenuta nel can. 1261, comporta effetti diversamente graduati e riferiti a differenti livelli.

Con riferimento all'ambito dell'agire dei fedeli nella società civile, considerato come livello esterno dell'agire, questa previsione si inserisce nell' ampia tutela che nell'ordinamento statuale è assegnata ai diritti civili e, quindi, non può ritenersi lecita l'attività dello Stato, quando tenda ad ostacolare e limitare o impedire gli atti spontanei devolutivi dei fedeli a beneficio della comunità ecclesiale. Al contrario, in linea con la disciplina civile del fenomeno religioso si presenta la complessa (quanto dinamica) regolamentazione dei benefici, delle sovvenzioni e delle liberalità riguardanti la comunità ecclesiale $(e$, in genere, le confessioni religiose), specie per quel che riguarda la rilevante innovazione in base alla quale è possibile dedurre dal reddito individuale le erogazioni in danaro a favore dell'istituto per il sostentamento del clero, unitamente alla destinazione a scopi di carattere religioso di una quota percentuale dell'imposta sul reddito delle persone fisiche, così come previsto in Italia a seguito dell'Accordo del 1984 modificativo del Concordato tra Stato e Chiesa. Anche con riferimento alla legislazione della Polonia, le novità introdotte con la legge n. 154 del 17 maggio 1989 nella parte terza relativa alle "Questioni patrimoniali delle persone giuridiche ecclesiastiche" ha determinato significativi cambiamenti nella vita patrimoniale e nell'attività degli enti ecclesiastici e delle stesse chiese, richiedendo comportamenti conseguenziali anche nella vita dei fedeli.

Con riferimento al livello interno, premesso che la norma fa riferimento a tutti i beni temporali e, quindi, non solo a quelli economici in senso stretto, si renderà necessario comprendere le linee di differenziazione con il successivo can. 1262, che disciplina i beni economici ecclesiastici. Inoltre, evidente è la relazione tra il can. 1261 e il can. 1267, in particolare con riguardo al pr. 3. La previsione del can. 1261, dunque, ha valore di limite all'attività degli amministratori dei beni ecclesiastici, i quali sono obbligati a rispettare la specifica destinazione delle liberalità del fedele. L'ampia formulazione del canone in parola sottolinea il carattere dell'unità e universalità della Chiesa, che deve equilibrarsi con il carattere più strettamente comunitario dell'esperienza dei christifideles. Pur nella legittimità degli spazi che in altre norme vengono riservati, anche nella subiecta materia, alle 
strutture di base-della comunità ecclesiale o ai movimenti e ad altre forme associative, l'indicata previsione normativa privilegia, nella sua impostazione generale, la struttura più ampia della Chiesa diocesana, nazionale, universale.

Sotto questo profilo è possibile intendere una ulteriore innovazione introdotta con riguardo peculiare, anche se non esclusivo, al ruolo delle Conferenze Episcopali e ad un rinnovato esercizio della potestas iurisdictionis episcopi. L'insistenza sul carattere ecclesiale delle imposizioni di diritto pubblico e, conseguentemente, sugli obblighi e sui diritti-doveri dei singoli Vescovi (come ad esempio: l'obbligo sancito dal can. 1261 pr. 2 di ammonire i fedeli quanto alla necessità obbligante di osservare il disposto del can. 222; il diritto di imporre contributi e tasse straordinarie, previsto dal can. 1263; i poteri di un controllo sia pure non del tutto libero sulle attività di amministrazione ordinaria, can. 1281 pr. 1, e sulle alienazioni, cann. 1291 e 1292) nonché su taluni poteri delle Conferenze Episcopali (ad esempio in materia generale sui tributi, can. 1262, sulle questue, can. 1265 pr. 2, sulle trasformazioni del sistema beneficiäle, can. 1272, sulla alienazione dei beni, can. 1292, sulla locazione dei beni, can. 1279) risponde altresì al bisogno di programmare l'uso e l'amministrazione dei beni ecclesiastici, ed in particolare le entrate patrimoniali della comunità ecclesiale, individuando linee progettuali, capaci di esprimere e concretare le finalità, di cui al can. 1254 pr. 2. Le previsioni del codice di diritto canonico consentono di individuare da un lato l'esistenza di linee di politica patrimoniale ecclesiastica, che si riferiscono all'acquisto dei beni da parte della Chiesa, e dall'altro lato di osservare l'importanza delle decisioni collegiali in materia. Infatti, spetta alla Conferenza Episcopale dettare norme riguardanti le imposte, cioè quegli atti di liberalità-dovuta connessi con le necessità della Chiesa per il perseguimento dei fini propri e, in definitiva, del fine ultimo di tutto l'ordinamento, cioè la salus animarum. La stessa Conferenza Episcopale ha il potere di stabilire le tasse (cioè il corrispondente dovuto per una prestazione ecclesiastica) per gli atti di potestà esecutiva graziosa, autorizzazioni, ammissioni, concessioni, approvazioni o per l'esecuzione dei rescritti della Santa Sede nonché determinare le offerte per l'amministrazione dei sacramenti, can. 1264. Infine, è nel potere della Conferenza Episcopale stabilire norme circa le questue e le collette, vincolanti per tutti $i$ fedeli, anche per quelli che scelgono uno stato di vita che comporti istituzionalmente la mendicità, can. 1265 pr. 2, 
poiché nell'esperienza della Chiesa la povertà è strumento di perfezione e non fine di vita.

Nell'ambito di queste norme, che complessivamente disciplinano la politica finanziaria della Chiesa, una collocazione peculiare spetta al diritto e alla funzione del Vescovo. Invero, per quel che riguarda l'acquisto dei beni e con riferimento alla imposizione di carattere pubblico, il can. 1263 stabilisce il diritto del Vescovo diocesano di imporre alle persone giuridiche pubbliche, che gli sono soggette, un contributo per le necessità della diocesi, che sia" non eccessivo ", e proporzionale al reddito delle stesse persone giuridiche.

L'imposta sulle persone giuridiche pubbliche non ha carattere personale (il nuovo codex non contempla ipotesi di imposizione in segno di sottomissione, come era ad esempio per il vecchio cathedraticum) né straordinario, bensi è direttamnte connessa con un bisogno ecclesiale „, pro dioecesis necessitatibus „. Tuttavia, il tributo può essere imposto solo previa consultazione del consiglio per gli affari economici e del consiglio presbiterale. La motivazione pastorale e il carattere ecclesiale dell'imposta emergono chiaramente dalle previsioni normative, tanto vero che il legislatore codiciale ha avvertito il bisogno di stabilire, indipendentemente dall'esito del parere, la consultazione previa di due organi che assolvono a funzioni differenti nell'ambito della organizzazione ecclesiastica, ancorché trattisi di funzioni partecipi dell'attività di governo del Vescovo.

La seconda parte del can. 1263, al contrario, disciplina una ipotesi di imposizione straordinaria nei confronti delle persone fisiche e giuridiche private, consentita solo ed esclusivamente ,in casu gravis necessitatis „e, comunque, sempre previa consultazione del consiglio per gli affari economici e del consiglio presbiterale. Sicché, dato il carattere straordinario della imposizione, è lecito ritenere che detta previsione contenga una proibizione di carattere generale che, logicamente correlata con l'importanza fondamentale che nella vita della Chiesa assume l'atto oblativo spontaneo da parte dei christifidele, impedisce la tassazione straordinaria delle persone fisiche e giuridiche private,

8 - Le oblazioni e le offerte sono, nella prospettiva del nuovo codex, largizioni fatte dai fedeli ,, ad certum finem ,, can. 1267 pr. 3 , tra quelli indicati nel can. 1254 pr. 2, senza che si determinino effetti giuridici successivi. In modo specifico vengono disciplinate le offerte effettuate per l'amministrazione dei sacramenti e dei sacramentali 
(cann. 848, 1264 pr. 2, 1181) e le oblazioni date per la celebrazione della messa. Quest'ultimo tipo di offerte, dal peculiare carattere sinallagmatico, trova una compiuta disciplina nei cann. 945-958 e risponde al fondamentale bisogno del sostentamento del clero e delle opere di carità, attuato secondo modalità di controprestazione „ut intentionem Missam applicet", can. 945 e cari. 946, escludendosi qualsivoglia intento di contrattazione o di commercio. Si tratta, quindi, di una peculiare sollecitudine da parte dei fedeli, attuata con modalità controprestative che tuttavia non sono obbligatorie, ben potendo mancare (can. 945 pr. 2) o risultare di modesta entità (can. 948) senza per questo che venga meno nel sacerdote l'obbligo alla celebrazioni della Messa, secondo l'intenzione proposta dai fedeli.

La delicatezza della materia, che giustamente viene trattata nel $\mathrm{Li}$ bro IV, „De Ecclesiae munere sanctificandi”, Parte I, „De Sacramentis", ha suggerito una disciplina prudente e precisa. Invero, le oblazioni per la Messa costituiscono una parte quantitativamente rilevante - se non la più rilevante - delle entrate patrimoniali della Chiesa; e, specie in passato, non pochi sono stati i contrasti e le crisi di comunione sul punto, a causa delle modalità, talora ,conimerciali", con cui veniva gestita l'offerta. La necessità di maggior rigore e trasparenza, a tutto privilegio della realtà sacramentale, esigenza da più parti espressa nella comunità ecclesiale, ha trovato accoglienza nel codex, che, anteponendo a tutto il valore sacramentale delia Messa, equilibra il carattere oblativo, partecipe della sollecitudine ecclesiale e finalizzato al sostentamento dei ministri sacri e delle opere di carità, con i controlli comunitari, di tipo gerarchico (confronta i cann. 957, 956, 958, 952).

9 - All'interno di questo grande ambito delle entrate patrimoniali si colloca la disciplina relativa ai contratti e alle alienazioni, Titulus $I I I$, e alle pie volontà e pie fondazioni, Titulus IV, che possono essere ricomprese nei modi di acquisto iure privatorum. La collocazione in due Titoli separati, verosimilmente 5 e fatta in ragione della peculiarità delle materie disciplinate, che - riferito al più generale diposto del can. 1259 in base al quale la Chiesa, concepita nel suo aspetto organizzativo e, pertanto, -nella pluralità delle istituzioni che da essa dipartono, acquista il potere di dominio sui beni temporali come ogni altro fenomeno entificativo soggetto di diritto - non presentano novità di rilievo. 
a) Per quel che riguarda i contratti, si può affermare che, in linea generale la disciplina è ispirata a prudenza specie con riferimento alle alienazioni, sulle quali è previsto il controllo autorizzativo dell'autorità ecclesiastica competente, can. 1291 , che si estende, in caso di mancato rispetto della formalità autorizzativa, fino all'esercizio di una azione, nel campo ecclesiastico e nel campo civile, ,ad Ecclesiae iura vindicanda ,, can. 1298 , che propone un contenuto più ampio rispetto a quello del can. 1534 del vecchio codex. Il can. 1279, innovando completamente rispetto alla vecchia normativa, introduce una ipotesi di intervento normativo collettivo, attribuito alla Conferenza Episcopale, circa la locazione dei beni della Chiesa.

Il contratto di locazione dei beni immobili della Chiesa, come è dato rilevare dagli eventi soprattutto recenti della storia, sovente non risponde all'ottica acquisitiva, poiché detti beni immobili vengono utilizzati per ospitarvi opere di carità e di apostolato. Sicché, devesi ritenere che la fattispecie disciplinata da detto canone più utilmente vada a collocarsi in relazione alle esigenze di politica ecclesiale generale circa l'utilizzo dei beni, rispondendo, quindi, ad un preminente obiettivo pastorale,

Nel can. 1290 è normativizzata la canonizzazione delle leggi dell'ordinamento civile dal quale vengono mutuati i principi che disciplinano la multiforme materia contrattuale. Il quadro generale delle finalità, che orientano il diritto patrimoniale della Chiesa, esclude qualsivoglia ipotesi di lucro, sia diretta che indiretta. In questa prospettiva il legislatore codiciale ha disposto nel can. 1294 par. 2 la possibilità di un investimento a beneficio della Chiesa o secondo le finalità dell' alienazione, con il ricavato. La cautela, che deve ispirare gli investimenti, e la prudenza da adottare nell'uso degli stessi secondo le finalità dell' alienazione sono una riprova della assoluta esclusione dello scopo di lucro e della necessità di ricondurre ad una valenza esclusivamente ecclesiale le attività disciplinate dal canone.

b) Per quanto riguarda le pie volontà (atti dispositivo, per atto tra vivi o mortis causa, dei beni per volontà specifica del disponente destinati ad un fine ecclesiale, di natura cultuale o caritativa o di apostolato) non risultano introdotte peculiari novità nella disciplina complessiva, ordinata nei cann. 1299-1310 in uno con le pie fondazioni (masse patrimoniali o di beni temporali suscettibili di essere autonomamente eretti in persona giuridica - le fondazioni pie autonome - destinate ad opera di pietà, di apostolato o di carità sia spiri- 
twale che temporale, come si evince dalla specifica disciplina nei cann. 1303-1307).

10 - Le novità più significative risultano introdotte nel Titulus II, relativo all'amministrazione dei beni.

L'esame globale delle norme contenute in questo Titolo, anche riferito in una visione di insieme alle altre disposizioni che disciplinano la materia dei beni temporali, consente di individuare, nella traccia delle opzioni conciliari e dell'immagine emergente di Chiesa nell'indicato quadro teologico di riferimento, talune linee generali, che, a mo' di principi direttivi presiedono all'amministrazione dei beni: a) la titolarità dell'amministrazione; b) la corresponsabilità nell'amministrazione; c) la complementarietà nell'amministrazione; d) la solidarietà e la mutua cooperazione; e) l'ordinaria e la straordinaria amministrazione.

a) In linea ordinaria, a mente del can. 1279 par, I, interpretato anche alla luce dei cann. 1256 e 118, l'amministrazione dei beni ecclesiastici spetta a colui che regge (ricomprendendo nella fattispecie anche ipotesi di reggenza collegiale) o rappresenta legalmente 1'ente, cui appartengono i beni; in tal modo si viene a stabilire un nesso tra la proprietà, la potestas regendi (in senso lato), e l'amministrazione. Detto nesso esprime da un lato il carattere di ministerialità specifica applicata ai beni ecclesiastici, che comporta la potestas regendi, dall'altro la necessità che anche le attività di amministrazione devono partecipare- delle stesse finalità che connotano il diritto di proprietà della Chiesa e nella Chiesa, come del resto espressamente stabilisce il can. 1254.

L'amministrazione dei beni ecclesiastici - cioè ,, l'insieme degli atti e delle attività che vengono compiute intorno a un bene o a un complesso di beni per acquisirli, curarne la conservazione e il miglioramento, percepirne ed erogarne i frutti e i redditi, e, infine, per alienarli „, (Rovera, I beni temporali della Chiesa, in La normativa del nuovo codice, Brescia, 1983, 271) - postula, quindi, un ministero finalizzato non al bene, bensì al diretto vantaggio della comunità ecclesiale, in modo che il bene economico consenta il perseguimento dei fini propri della Chiesa. Essa, inoltre, comporta il porre in essere di una serie di atti e attività, elencate a titolo esemplificativo nel can. 1284 par. 2, che devono essere esperite con l'ordinaria ,, diligente boni patris-familias ", di cui al can. 1284 par. 1, partecipazione ed esplicitazione peculiare dell'imprescindibile sensus Ecclesiae. E invero, a mente del can. 1282 , tutti coloro che hanno parte all'amministrazione dei beni eccle- 
siastici spendono l'immagine della Chiesa in una sorta di contemplatio domini, fondata sulla volontà del legislatore. L'agire in nome della Chiesa, lungi dall'essere considerato alla stregua di un negozio di diritto privato, ha prima di tutto effetti nella sfera etica e morale e sottolinea la dimensione, per così dire, pubblica, cioè riferita alla comunità ecclesiale, dell'attività dell'amministratore dei beni ecclesiastici, appartenenti, quindi, alle sole persone giuridiche pubbliche, volta al perseguimento del bene comune.

In questa prospettiva, il ministero del Romano Pontefice, disciplinata dal can. 1273 e, in via incidentale, dal can. 1256 è di duplice natura: da un lato egli è vero e proprio amministratore, potendo compiere atti e attività direttamente riferite a beni ecclesiastici, dall'altro, per la condizione di parità amministrativa dettata dal can. 1282, agendo tutti gli amministratori con responsabilità propria, personale e diretta (in tal senso cfr. i cann. 1283, 1284, 1287, 1289), limitatamente all'amministrazione dei beni ecclesiastici, esercita funzioni di controllo e tutela sul patrimonio ecclesiastico e sull'attività dell'amministratore.

Parallele considerazioni possono farsi per quel che riguarda il ministero del vescovo circa l'amministrazione dei beni ecclesiastici. Per quel che riguarda, tuttavia, l'ipotesi dell'amministrazione diretta, il codice contempla una potestà diretta solo limitatamente ad alcuni beni, deducendosi ciò sia dalla previsione generale del can. 1279, secondo la quale l'amministrazione diretta spetta solo a chi regge la persona cui $\mathrm{i}$ beni appartengono, sia dalla specifica statuizione del can. 1274 par. 3, secondo la quale a carico del Vescovo è posto l'onere di provvedere agli obblighi verso le persone che servono la Chiesa per il tramite del fondo comune.

Pertanto, l'ordinaria attività di amministrazione che compete al vescovo è orientata da un duplice compito: il primo di vigilanza e controllo sull'amministrazione dei beni delle persone giuridiche pubbliche che gli sono sottoposte, secondo il combinato disposto dei cann. 1276 par. 1, 1279, 1281, che concreta l'esercizio di una potestas, preventiva e successiva sugli atti e comportamenti specifici dell'amministrare, nell'interesse della comunità ecclesiale; il secondo di carattere direttivo, a mente del can. 1276 par. 2, in virtù del quale la legge gli riconosce un potere pubblico generale, sempre a vantaggio della comunità ecclesiale, di disciplina dell'amministrazione nell'ambito della propria diocesi, esercitato con la predisposizione di norme e istruzioni, caratterizzate dal peculiare oggetto unificante e dall'organico 
inserimento, secondo una visione generale e coordinata, nelle finalità proprie della comunità ecclesiale, richiamate nel can. 1254 par. 2.

Queste previsioni consentono di scorgere una attuazione, ancorché circoscritta e delimitata, del principio di sussidiarietà, ispirato al precetto stabilito nel par. 16 del decreto conciliare Chritus Dominus, dettato circa il dovere di governare dei pastori.

b) Altro principio direttivo, derivato dalla concezione di Chiesa-comunità che presiede alla legislazione codiciale, è quello della corresponsabilità. Essa si esprime soprattutto per il tramite della istituzione di nuovi organismi, collegiali e individuali, il cui potere di intervento circa l'amministrazione dei beni ecclesiastici è diversamente regolato.

La previsione di carattere generale, dettata nel can. 1280, istituisce il consiglio per gli affari economici, il cui compito fondamentale è quello di coadiuvare l'amministratore nell'esercizio del suo ministero. Detto canone, che presenta un carattere di precettività, per così dire, ritardata per il necessario adattamento alle differenti realtà ecclesiali nelle quali operano le varie persone giuridiche pubbliche, trova una peculiare attuazione con riferimento alla comunità diocesana $\mathrm{e}$ alle parrocchie che ad essa afferiscono. Infatti, per quel che riguarda la diocesi norme precise circa l'istituzione, i compiti e il funzionamento del consiglio per gli affari economici vengono dettate nei cann, 492 e 493: questa specifica disciplina prevede l'obbligatoria partecipazione al consiglio della predisposizione del bilancio preventivo delle questue e delle elargizioni e l'approvazione del bilancio consuntivo delle entrate e delle uscite. La previsione consente di rilevare una estensione - a detto organismo della potestas direttiva in materia di amministrazione dei beni ecclesiastici, attribuita alla persona del vescovo. Sicché, la particolare normativa dettata per la Chiesa diocesana sottolinea il rilievo pubblico dell'amministrazione, come atto di chiesa. Eguale considerazione può farsi per quel che riguarda il consiglio parrocchiale per gli affari economici, disciplinato in modo particolare dal cari. 537, a mente del quale il vescovo diocesano ha poteri regolamentari integrativi del diritto universale nel dettare le norme che presiedono l'attività di detto organismo.

In linea generale, nell'interpretazione letterale e sistematica delle indicate disposizioni, specie tenendo conto della collocazione nel contesto dei Libri del codex (il consiglio diocesano per gli affari economici è disciplinato nel Liber II, „De populo Dei”, Pars II „, De Ecclesiae constituzione hierarchica”, Sectio II, „De Ecclesiis 
particularibus deque earundem coetibus”, Tit. III, „De interna ordinatione Ecclesiarum particularium"), si rileva un diretto riferimento alla comunità e un ambito specifico di esercizio della corresponsabilità estesa a tutti i christifideles. Detta corresponsabilità, che è condizione riferita a tutto il popolo, non ha carattere esclusivo (confronta in tal senso il generale disposto del can. 1282 nella parte iniziale: $<$ Omnes, sive cierici sive laici, qui legitimo titulo partes habent in administratione... >>) $\mathrm{e}$, in più, non è meramente eventuale e facoltativa. Invero, in taluni adempimenti essa è necessaria ed è considerata condizione di legittimità dell' attività dell' amministratore (come nella ipotesi prevista dal can. $1281 \mathrm{pr} .2$ per la previa attivazione del consiglio per l'individuazione degli atti di straordinaria amministrazione delle persone soggette alla potestà del vescovo, e nella ipotesi prevista dal can. 1277 , secondo il quale il vescovo non può porre atti di ordinaria amministrazione di peculiare importanza per la diocesi se non previa consultazione del consiglio per gli affari economici e del collegio dei consultori).

E' pur vero, tuttavia, che questa generale previsione di corresponsabilità, a parte alcune ipotesi di obbligatoria consultazione, pur dovendosi ritenere estesa a tutti gli atti e le attività dell'amministrare, è proporzionale al sensus ecclesiae dell'amministratore, al quale è rimessa la discrezionalità degli ambiti entro i quali consentirne l'esercizio.

Nella linea della corresponsabilità va interpretata la normativa che istituisce un altro organismo, il collegio dei consultori. Detto organismo -istituito nel can. 502, che disciplina le funzioni, la durata e il numero dei componenti- per quel che riguarda l'amministrazione diretta ha compiti circoscritti e delimitati. In realtà, il collegio ha compiti che più direttamente si riferiscono al governo della diocesi (in tal senso ha anche assorbito talune funzioni che il vecchio codex attribuiva al capitolo), i quali derivano dal legame alla persona del vescovo, che lo presiede, e al consiglio presbiterale, all'interno del quale è costituito. In tale prospettiva, l'obbligo (che non pare possa essere considerato come vincolante) della consultazione del collegio per gli atti dell'amministrazione insorge ogni qualvolta la decisione da adottare comporti l'esercizio di una potestà riferita alla più ampia funzione di governo della Chiesa locale. Se, poi, le attività dell'amministrazione sono di carattere straordinario e collocate sempre all'interno dell'ampia potestà di governo diventa necessario, oltre alla consultazione, il consenso sulle decisioni da prendere, indicate dal vescovo. 
In un senso analogo va altresì intesa la corresponsabilità del consiglio presbiterale, applicata all'amministrazione dei beni ecclesiastici. Infatti, interpretando in modo combinato il disposto dei cann. 495, 500 pr. 2, 1263, si può individuare una funzione consultiva del consiglio in materia di beni ecclesiastici in quelle ipotesi nelle quali la loro amministrazione comporti un nesso con l'attività di governo per il bene pastorale della comunità ecclesiale. Questa funzione diventa necessaria e obbligatoria nell'ipotesi di imposizione contributiva ordinaria posta per le necessità della diocesi, senza, tuttavia, che il parere del consiglio presbiterale, reso quale partecipazione all'ampio munus di governo, risulti vincolante.

Una osservazione a parte merita il consiglio pastorale diocesano. Infatti, la disciplina circa i beni ecclesiastici non prevede alcuna ipotesi di coinvolgimento di questo organismo. L'omissione sembra partecipare di quella più ampia precarietà istitutiva dettata dal can. 511, che condiziona apoditticamente la costituzione del consiglio pastorale diocesano ad una valutazione e ad un apprezzamento di convenienza del Vescovo. Tuttavia, al di là dello stretto dettato normativo e tenendo conto della competenza del consiglio circa le attività pastorali della diocesi, ove fosse costituito, ben potrebbe ipotizzarsi l'esercizio di una corresponsabilità affievolita, perché rimessa ad un mero atto episcopale e alla natura consultiva del voto, specie in considerazione della valenza ecclesiale e, quindi, pastorale della disciplina codiciale circa i beni ecclesiastici.

Altra figura rilevante è quella dell'Economo, assolutamente nuova e disciplinata dai cann. 494 e 1278. Il codice gli attribuisce ampi compiti di amministrazione ordinaria e straordinaria dei beni della diocesi <<sotto l'autorità del Vescovo >, ma secondo le <<modalità definite dal consiglio per gli affari economici > (can. 494 pr. 3). Il duplice vincolo che orienta l'attività dell'economo, al quale possono essere affidati in amministrazione dallo stesso Ordinario anche $i$ beni delle persone giuridiche pubbliche a questi sottomesse (can. 1276 pr. 1 e can. 1279 pr. 2), anche in relazione alla distinzione che dalla normativa è desumibile tra beni della diocesi e beni delle persone giuridiche pubbliche, induce a sottolineare la peculiare funzione ecclesiale di tale ufficio (in tal senso va letta la soggezione all'autorità del vescovo) in una indispensabile programmazione economica generale, che si concreta nelle modalità indicate dal consiglio per gli affari economici. Questi due elementi, che contestualmente ed inscindibilmente caratte- 
rizzano l'attività amministrativa dell'economo, spiegano altresì il particolare regime stabilito per la nomina - in vista della quale obbligatorio, sebbene non vincolante, è il parere del collegio dei consultori e del consiglio per gli affari economici (can. 494 pr. 1)- e per le ipotesi di rimozione dall'ufficio, che ha comunque durata quinquennale (can. 494 pr. 2). Al contempo, è individuabile a carico del vescovo un duplice potere di vigilanza: un primo di carattere generale e personale, che discende dalla peculiare sottomissione dell'economo alla sua autorità, ed un secondo potere specifico concernente l'osservanza, da parte dell'economo, di quelle modalità determinate dal consiglio per gli affari economici all'interno di quelle necessitate linee di politica economica, alla quale deve essere orientata l'amministrazione dei beni nella Chiesa diocesana.

c) Al principio della complementarietà nell'amministrazione dei beni possono essere ricondotti quegli istituti di nuova concezione, i quali possono meglio concretare ed evidenziare la natura carismatica del servizio ecclesiale a tutto beneficio di una Chiesa che si concepisce come comunità: l'istituto speciale di raccolta dei beni e delle offerte, il fondo diocesano comune.

L'attenzione alla complementarietà, dimensione fondamentale per la fondazione della comunità cristiana (richiamata nelle diverse circostanze dai vari episcopati nazionali, come ad esempio quello italiano che ne ha fatto menzione sin dal piano pastorale per gli anni ' $80 \mathrm{Co}$ munione e Comunità, nn. 65 e 66), consente di esprimere più autenticamente il ministero personale, che è partecipazione del ministero profetico, sacerdotale e regale di Cristo, inserito in un rapporto di necessarietà e di organicità con altri ministeri personali, condizione unica per la realizzazione dell'unità ecclesiale. D'altro canto, il fenomeno della integrazione dei ruoli trova una peculiare espressione al livello delle relazioni tra le Chiese particolari, la cui varietà $<<$ dimostra con maggiore evidenza la cattolicità della Chiesa indivisa $>$ (cfr. $\mathrm{Lu}$ men Gentium, 23).

In questa prospettiva possono essere compresi i due istituti sopra indicati e disciplinati dal can. 1274.

L'istituto speciale di raccolta di beni e offerte per il sostentamento del clero, che dopo l'eliminazione del beneficio - secondo il dettato del can. 1222 - costituisce lo strumento economico principale per l'attuazione della perequazione economica del clero, trova la ragione dell'esistenza nell'attività ministeriale del sacerdote, al quale - a mente 
del can. 28 1, richiamato dal can. 1274 - spetta una remunerazione rapportata alla condizione personale, alle circostanze di tempo e luogo, alla natura dell'ufficio e proporzionata alle necessità della vita e all'eventuale onere derivante dalla giusta retribuzione di eventuali collaboratori domestici.

Proprio il rivelato legame tra le due disposizioni, coordinate tra di loro in virtù dell'importanza che il legislatore intende conferire al ministero che svolgono i presbiteri (piuttosto che allo status del sacerdote), mette in evidenza il rapporto tra ministero e servizio alla comunità. In tale prospettiva il perseguimento di una delle tre finalità indicate nel cari. 1254 pr. 2, e cioè il sostentamento del clero, non acquista valenza come problema di categoria, bensì come questione ecclesiale. Pertanto, il complesso rapporto interpersonale -strutturato in un ineliminabile rapporto di comunione, che giustifica e fonda l'insostituibilità del singolo battezzato nel popolo di Dio- richiede l'esistenza di ministeri, costituiti a beneficio di tutta la comunità (cfr. Lumen Gentium nn. 71, 18,22), e il conseguente insorgere di obblighi e diritti.

Eguale dinamica va rilevata nellä disposizione del par. 3 del can. 1274 , che disciplina la costituzione del fondo diocesano comune. E' opportuno ricordare che il fondo comune è previsto per il perseguimento di tre obiettivi equivalenti: il soddisfacimento degli obblighi verso persone che hanno un ufficio nella Chiesa, per andare incontro alle necessità della diocesi, per l'aiuto delle diocesi più povere. La valenza ecclesiale degli obiettivi per la costituzione del fondo comune, quindi, coinvolge due livelli: uno interno alla diocesi ed uno esterno, afferente le relazioni tra le varie Chiese particolari. Quest'ultimo aspetto consente di meglio cogliere il salto di qualità compiuto quando si è preferito adottare l'opzione conciliare circa l'immagine di popolo. Infatti, la categoria di popolo esprime la moltitudine dei fedeli, che, nelle comunità ecclesiali particolari, operano per il raggiungimento del fine dell'unica e unita Chiesa cattolica.

Sicché, il fondo dovrà rappresentare un solo istituto, sia pure con bilanci e/o contabilità distinte laddove se ne rilevi la necessità, e mostrare omogeneità di intervento in riferimento agli scopi specifici, indicati nel can. 1274 pr. 3.

d) Il principio della solidarietà, comprensivo anche dell'aspetto della mutua cooperazione, sembra orientare la disciplina degli istituti diocesani federati, previsti dal can. 1274 pr. 4 , per una più congrua attuazione delle finalità circa il sostentamento del clero e per una più 
efficace operatività del fondo comune. Nella stessa prospettiva può essere interpretato il can. 1275, il quale prevede che quando il fondo dei beni proviene da diocesi diverse venga amministrato secondo una normativa comune $<<$ concordata dai Vescovi interessati $>$. Nell'indicata normativa viene ad evidenza la dimensione, per così dire, esterna e cattolica della solidarietà, in virtù della quale le relazioni tra le Chiese particolari vanno reimpostate in una prospettiva destinata a superare il circoscritto territorio diocesano, che trova una propria autentica espressione allorquando si proietta nella Chiesa universale, costruita dalla comunione delle singole comunità ecclesiali. Tale rinnovata dimensione missionaria del popolo di Dio determina, pur nell'autonomia delle singole Chiese diocesane, un obbligo assunto in comune e una inscindibile corresponsabilità, sia in riferimento a situazioni e necessità interne sia in relazione alle istanze e alle aspettative di tutto il genere umano. La solidarietà è, quindi, espressione della comunione interecclesiale e fonte di comune attività e responsabilità per il conseguimento di specifiche ed attuali finalità.

e) Per quel che riguarda, poi, l'ordinaria e la straordinaria amministrazione, anche nel nuovo codice non vi è una chiara distinzione circa le attività da riferire di volta in volta nell'uno o nell'altro ambito, fatte salve incontestabili eccezioni (quali ad esempio gli atti di alienazione). Ciò consente di dare la giusta rilevanza alla prassi delle singole chiese diocesane e alla loro consuetudine amministrativa. Al contrario, vengono indicate rigorose formalità e precisi controlli che devono accompagnare lo svolgimento degli atti di straordinaria amministrazione, connotandone la liceità, la validità e la nullità, la responsabilità. Questo sistema, apparentemente, presenta elementi di contraddittorietà e non poche difficoltà pratiche, tuttavia è caratterizzato dalla generale esigenza di non compromettere in alcun modo la realizzazione delle finalità proprie, indicate nel can. 1254 pr. 2, e, di conseguenza, l'immagine stessa della Chiesa. Per questo, si può dire che, in genere, quegli atti e quelle attività di disposizione dei beni ecclesiastici, che, per il fatto di essere di grande importanza in riferimento alla concreta situazione economica generale (confronta la prima parte del can. 1277), comportano una sostanziale modifica della situazione di fatto e di diritto del patrimonio ecclesiale -sia della Chiesa diocesana sia di una persona giuridica pubblica- possono essere qualificate di straordinaria amministrazione e richiedono le solennità e le garanzie di formalità e sostanza previste dal diritto. Inoltre, il codice consente 
di introdurre una ulteriore distinzione, a mente del disposto del can. 1277 , fra atti e attività riferite ad affari di grande importanza economica relativa -che possono essere considerati a cavallo tra l'ordinaria e la straordinaria amministrazione- ed atti e attività di amministrazione straordinaria in senso proprio. Queste ultime devono essere espressamente indicate e qualificate come tali dagli statuti delle singole persone giuridiche pubbliche (can. 1277 e 1281 pr. 2) e dal Vescovo, sentito il consiglio per gli affari economici, limitatamente alle persone sottoposte alla sua autorità (can. 1281 pr. 2), dagli organismi di governo degli istituti religiosi (can. 638 pr. 1), dalla Conferenza episcopale (can. 1277). Particolari requisiti di forma, quelli previsti dai canoni dal 1291 al 1294, vanno osservati anche in ogni affare suscettibile di intaccare in senso peggiorativo il patrimonio della persona giuridica, secondo il disposto del can. 1295.

Una menzione a parte merita il can. 1285 , il quale, disciplinando le donazioni a fini di pietà e di carità, sia pure limitatamente ai beni mobili non appartenenti al patrimonio stabile in relazione ed entro i limiti della ordinaria amministrazione, öffre l'opportunità di evidenziare la grande importanza che nella vita della Chiesa assumono le opere di pietà e di carità, le quali, in linea generale, possono essere ricomprese nell'ordinaria amministrazione in quanto direttamente discendenti dalla sollecitazione evangelica alla fedeltà a Dio e agli uomini.

Infine, va rilevato il peculiare regime della responsabilità in riferimento alla produzione di atti e al compimento di attività di straordinaria amministrazione inficiati di invalidità e di illiceità: in linea generale, solo se dall'atto o dal comportamento invalido la persona giuridica trae vantaggio la responsabilità dell'amministratore si trasferisce alla persona giuridica. Ugualmente v'è responsabilità della persona giuridica per gli atti illeciti posti dagli amministratori, verso i quali, tuttavia, può essere iniziata una azione di responsabilità personale (can. 1281 pr. 3).

11 - L'esame che precede, volto a individuare taluni caratteri di novità $\mathrm{e}$ unità del regime riguardante $\mathrm{i}$ beni temporali della Chiesa, consente anche di introdurre una riflessione circa la necessità di reimpostare il rapporto con l'ordinamento civile nella subiecta materia.

Invero, l'ordinamento canonico conosce forme di interazione con gli ordinamenti civili quando e predispone meccanismi circoscritti di collegamento, come il fenomeno della canonizzazione delle leggi ci- 
vili (come ad esempio avviene nelle ipotesi di rinvio disciplinate dal can. 1286 pr. 1, circa l'osservanza delle leggi civili nei rapporti di prestazione d'opera da parte di amministratori di beni, dal can. 1290, circa l'osservanza delle norme civili nei contratti e nei pagamenti, dal can. 1299 pr. 2, circa le formalità del diritto civile da osservare in caso di disposizioni mortis causa) o più in generale il riferimento diretto al diritto statuale (come è nell'ipotesi disciplinata dal can. 1274 pr. 5, circa il riconoscimento agli effetti civili degli istituti speciali di raccolta dei beni e delle offerte). Tuttavia, non è possibile ignorare che, anche con riferimento alla materia dei beni, il rapporto tra Chiesa e Stato non si esaurisce nell'ambito delle relazioni interordinamentali, che appartengono al diritto pubblico esterno dell'ordinamento canonico.

Questo ambito è interessato dalle novità che sono state introdotte sia come applicazione interna dei concordati, che nel postconcilio la Chiesa ha stipulato con gli ordinamenti civili, sia come adattamenti unilaterali dell'ordinamento canonico rispetto ai mutamenti subiti dagli ordinamenti civili nella materia patrimoniale.

Tra i casi emblematici di questo fenomeno possono essere indicati quello dell'Italia e della Polonia, paesi nei quali i concordati hanno accompagnato i cambiamenti sociali e istituzionali, modificando in modo significativo i regimi giuridici precedenti.

Per esempio, per quanto riguarda l'Italia, il nuovo Accordo, siglato nel febbraio del 1984 ed entrato in vigore nel maggio del 1985, ha comportato un cambiamento radicale del rapporto tra Chiesa e Stato, improntato al riconoscimento bilaterale della sovranità e dell'autonomia, al rispetto della diversità dei rispettivi ordinamenti, alla individuazione di strumenti di cooperazione per il bene del Paese e il progresso dell'uomo. Ciò nella materia patrimoniale ha comportato l'abbandono di strumenti di carattere confessionista e la scelta di strumenti di laicità, che hanno favorito l'abolizione degli aiuti economici diretti dello Stato verso la Chiesa sostituiti da meccanismi di trasferimento di flussi finanziari determinati dalle scelte dei cittadini. Contemporaneamente l'ordinamento statuale ha introdotto modifiche sostanziali al proprio sistema impositivo e fiscale (anche in relazione all'ampliarsi dell'esperienza delle organizzazioni non profit e delle organizzazioni non lucrative, ONLUS) e alle regole che disciplinano il governo dell'economia, imponendo l'accelerazione dei processi innovativi avviati nell'ambito dei beni temporali. 
Il quadro delle relazioni tra società civile e società religiosa, che, in modo diretto o indiretto, riguardano la sfera dei beni temporali si va meglio individuando e precisando anche in conseguenza delle innovazioni (imposte da autonome necessità di rinnovamento della società civile) che l'ordinamento statuale progetta ed attua nella disciplina dei complessi settori della convivenza sociale. Tra le materie che si riferiscono a detto ambito di rapporti. tra società, e che non sempre presuppongono l'esistenza di una specifica disciplina concordata e normalizzata in un atto di diritto esterno, si possono menzionare quelle riguardanti la tutela del patrimonio artistico, la disciplina del volontariato e le convenzioni con enti autarchici, la pianificazione urbanistica per la costruzione di edifici di culto, le istituzioni di pubblica assistenza e beneficenza, l'esercizio della solidarietà nelle ipotesi di calamità naturali o di protezione civile. L'ambito sempre più ampio delle ipotesi di incontro e cooperazione tra società civile e società religiosa, con riguardo alle realtà temporali, comporta che l'attuazione della disciplina circa $\mathrm{i}$ beni temporali stabilita nell'ordinamento canonico tenga conto anche dei riflessi che i cambiämenti sociali e normativi prodottisi nell'ambito statuale hanno comportato nelle relazioni tra ordinamento canonico e ordinamento statale.

Per quel che riguarda la Polonia, il Concordato del 28 luglio 1993, ratificato il 25 marzo del 1998, ma solo di recente materialmente applicato, impone un complessivo ripensamento dei modi di gestione delle attività ecclesiali e della stessa vita, per così dire, economica della Chiesa, poiché assumono rilevanza bilaterale i prevedibili cambiamenti riguardanti le questioni finanziarie degli enti e delle istituzioni ecclesiastiche e il sostentamento del clero, tanto che è stata prevista la creazione di una commissione mista per i necessari comuni provvedimenti, e soprattutto è riconosciuta la competenza statale per la regolamentazione delle questioni finanziarie e patrimoniali (art. 22).

Tenendo conto, quindi, delle modificazioni sociali e giuridiche che si sono prodotte, si deve osservare che l' uso e l'amministrazione dei beni ecclesiastici sempre più diventa espressione della testimonianza di una comunità ecclesiale, che concepisce le finalità per le quali si riconosce il diritto nativo di acquistare, possedere, amministrare e alienare $\mathrm{i}$ beni temporali come orientate verso una peculiare proiezione sociale delle opere di apostolato e di carità, specialmente a servizio dei poveri (cfr. can. 1254). 